\title{
Análisis de la situación laboral del profesorado de Ciencias de la Comunicación en España
}

\author{
Javier SIERRA SÁNCHEZ \\ Universidad San Pablo CEU \\ jsierra@ceu.es
}

Resumen:

En el presente trabajo llevamos a cabo una aproximación analítica sobre la situación laboral y académica del profesorado de Ciencias de la Comunicación en España. Pretendemos conocer cuál es la situación real del profesional que imparte docencia e investiga en los grados de Publicidad y RR.PP., Comunicación Audiovisual y Periodismo. Con este macroobjetivo hemos diseñado un cuestionario que, a través del análisis de variables como la categoría profesional, la remuneración salarial o el número de créditos impartidos, entre otras, nos permita obtener una radiografía sobre el desempeño actual del profesor universitario, así como detectar las principales dificultades y retos a los que se enfrenta este colectivo.

Palabras clave: profesor; Ciencias de la Comunicación; situación laboral; carrera docente.

\section{Analysis of the employment situation of the Communication Sciences teaching staff in Spain}

\begin{abstract}
:
In this work, we carry out an analytical approach to the employment and academic situation of the Communication Sciences teaching staff in Spain. Our aim is to discover the real situation of professionals who teach and carry out research in the Advertising and Public Relations, Audiovisual Communication and Journalism degrees. With this macro-objective, we have designed a questionnaire that analyses variables such as professional categories, wages or the number of credits given, among others, thus enabling us to obtain a complete picture about the current performance of university professors, as well as to detect the main difficulties and challenges that this group faces.
\end{abstract}

Key words: professor; Communication Science; employment situation; teaching career.

\section{Referencia normalizada:}

Sierra Sánchez, J. (2014): Análisis de la situación laboral del profesorado de ciencias de la comunicación en España. Historia y Comunicación Social. Vol. 19. Núm. Especial Febrero. Págs. 759-778.

Sumario: 1. Introducción. 2 Objetivos. 3. Metodología. 4. resultados: descripción y análisis. 5. Conclusiones

\section{Introducción.}

La aprobación del Real Decreto 1393/2007, de 29 de octubre, por el que se establece la ordenación de las enseñanzas universitarias oficiales supuso la puesta en marcha del Plan Bolonia en España. Esta reforma educativa ${ }^{1}$, con el alumno como 
protagonista de su propio aprendizaje, afectaba a aspectos tan fundamentales como la movilidad e intercambio de alumnos, el aprendizaje basado en competencias, la estructuración de las titulaciones y las materias en créditos europeos a través del European Credit Transfer System (ECTS) ${ }^{2}$, la reducción del número de alumnos por aula, etc. Torrego (2004:263) detecta la escasa alusión que se hace al profesorado en los textos oficiales de "convergencia europea" a pesar de que, a nuestro juicio, la eficacia de la mayor parte de los cambios que propone dicha reforma necesitan para su implantación del compromiso y labor de los docentes.

Existe cierto consenso en la premisa de que la universidad española necesita cambios, mejoras que incrementen la calidad de la investigación, de la docencia y, en definitiva, la excelencia en los resultados de aprendizaje de sus alumnos. Este será el único camino para que las universidades españolas puedan competir en un mercado, el de la educación, cada vez más globalizado.

Sin embargo, la discrepancia se plantea en el modo de conseguir que estos cambios sean una realidad y no constituyan una mera declaración de intenciones teórica. Una reforma integral del sistema universitario como el Plan Bolonia requiere necesariamente de un incremento en la dotación de recursos económicos y humanos que permitan consolidar el espíritu del Espacio Europeo de Educación Superior. Rodríguez Rojo (2000:91) insiste en esta necesidad y Salas Velasco (2010) apunta en este mismo sentido cómo la transposición que se hizo en España de la declaración de Bolonia no estuvo reforzada de la dotación económica adecuada:

¿Cómo es posible acometer una reforma de tal envergadura "a coste cero" como propugnan la mayoría de las universidades? Se pretende dar estímulos equiparables con Europa, pero los medios son muy distintos (infraestructuras, recursos electrónicos, ordenadores, etc). Los datos de la OCDE revelan un importante déficit en el nivel de gasto en enseñanza superior en España. (Salas Velasco, 2010.p.22)

Este autor (2010:27) se muestra escéptico sobre los cambios que puede suponer la introducción de España en el EEES. Considera que el mayor riesgo es que la transformación sea meramente cosmética y apunta los ejes sobre los que debería centrarse la reforma universitaria: 1) sistema de financiación de las universidades (búsqueda de alternativas); 2) situación de precariedad laboral del profesorado (bajo nivel salarial); 3) mejora de la productividad científica; 4) bajo nivel de esfuerzo y motivación del alumnado; y 5) una necesaria profesionalización de la gestión.

El proceso de convergencia europea presenta retos para el alumno, el profesor y la Universidad. Todos estos aspectos nos invitan a realizar una profunda reflexión sobre la misión que la Universidad debe cumplir en nuestra sociedad. Sin embargo, no todos los agentes que deben intervenir en dicha renovación han sido igualmente tratados ya que, con el foco de interés puesto en el aprendizaje del alumno, el desarrollo de un nuevo modelo organizativo para la institución universitaria y la redefinición de la función docente presentan importantes lagunas que no hacen sino plantear un futuro incierto para el sistema universitario resultante del Plan Bolonia. 
El EEES propugna un planteamiento docente en el que el alumno es el centro de un proceso de aprendizaje basado no sólo en la retención de conceptos teóricos sino en la adquisición de competencias y habilidades que le capaciten para la vida profesional. Este modelo de participación y aprendizaje cooperativo implica una redefinición de los planes de estudio, un incremento de infraestructuras y medios técnicos a disposición del alumno así como un importante aumento en las horas de dedicación del profesorado que, a su labor investigadora y docente, ha de añadir horas de trabajo destinadas a la labor tutorial individualizada, el diseño y corrección de ejercicios prácticos, la generación de contenidos on-line, tareas de gestión académica, etc.

Si el profesor es una pieza clave en la actividad universitaria tal y como apunta Ferreira (2010: 54) cuando insiste en el hecho de que la calidad de una universidad se mide por la excelencia de su profesorado, este se convierte en el auténtico vehículo para el cambio y solamente esta consideración puede constituir un punto de partida solvente para el mismo.

Decíamos que la reforma del Plan Bolonia persigue la calidad y la excelencia de los programas y de las Universidades. Con este objetivo, han proliferado las agencias de Calidad y el proceso de promoción del profesorado ha quedado supeditado a la acreditación de los méritos de la carrera académica en tres bloques: 1) docencia, 2) gestión e 3) investigación; primando este último apartado por encima del resto. Esta tripe función del profesorado comienza a ser considerada en la Ley Orgánica de Universidades (LOU) donde se crea la Agencia Nacional de Evaluación de la Calidad y la Acreditación (ANECA). Los procesos de acreditación del profesorado, en la práctica, alargan y complican la carrera docente universitaria ya que realizan una evaluación asimétrica de los méritos de docencia e investigación para cada una de las figuras de profesor contratado ${ }^{3}$, minimizando la importancia de la gestión académica e imponiendo un modelo de profesor que numerosas instituciones universitarias no pueden sostener. Si la producción investigadora es la vía principal para la acreditación del profesorado, la docencia y la gestión son las funciones con mayor dedicación requeridas por las universidades, sobre todo por las de titularidad privada.

Este marco estructural y legal y la consideración que hace de la labor investigadora de los docentes, tiene una consecuencia directa: los profesores se decantan por hacer méritos en su carrera académica relacionados con la investigación (publicación de artículos científicos, libros, participación en proyectos de investigación competitivos) en menoscabo de la docencia. Murray (1993) en Rodríguez Espinar (1994:43) ya alertaba sobre esta cuestión cuando planteaba que "el profesorado pondrá esfuerzo y talento en la mejora de la docencia, si la excelencia en la misma contribuye significativamente a la promoción y al incremento salarial". A esta reflexión es necesario añadir que en los procesos de selección del profesorado se priman los méritos de investigación por encima de la docencia, tal y como afirma Manjón (2000:53). De ahí que, como advierten Ferrer y González (1999: 330), la docencia sea percibida como una "compensación inevitable que se exige para poder ejercer la investigación y otras actividades en la Universidad". En este sentido, y como consecuencia de esta 
visión, estos autores señalan que la formación del profesorado se centra en cuestiones de índole pedagógica.

Esta desproporción estructural en la consideración de las funciones y valor del docente universitario, se ve además influida por otra circunstancia: la inestabilidad laboral. A pesar de que, como señala Feixas (2004: 45) "la precariedad contractual afecta negativamente al desarrollo de las tareas docentes e investigadoras del profesorado", este es un aspecto que no ha centrado especial atención en la reforma del sistema universitario. Además de la inestabilidad laboral, los sueldos de los profesores españoles están muy por debajo de la media europea. Rodríguez Rojo (2000:95) se refiere a los salarios cuando indica que "el salario medio de un profesor español en la universidad está en 27.025 dólares, mientras que el salario medio de la UE está en torno a 50.000 dólares". Esta misma situación es advertida por Salas (2010: 24) cuando insta a las Universidades españolas a moverse por una política de selección de profesorado con talento y enormemente exigente. Pero para tener los mejores profesores e investigadores los salarios deberían subir -las retribuciones actuales son bajísimas en comparación con universidades estadounidenses o inglesas- y los incentivos deben estar claros. En este mismo sentido, Knight (2005:23) afirma que "es imprescindible que (las personas que ejercen la docencia universitaria) estén satisfechas con sus niveles salariales, las perspectivas de promoción y el tratamiento, por el reto intelectual de la investigación, el placer de la enseñanza y la calidad emocional". Así Martínez-Otero (2010:10) pone de manifiesto la necesidad de mejorar las condiciones de los profesores asociados y contratados puesto que de continuar en esa situación de precariedad (un buen número de sus profesores) la Universidad no podrá cumplir la elevada misión que le ha sido encomendada.

Esta precariedad laboral caracterizada por salarios bajos e inestabilidad contractual se ve acrecentada por la asimetría existente entre los méritos requeridos por los procesos de acreditación y las funciones exigidas por las universidades a sus docentes, donde la gestión y la docencia ocupan la mayor parte de sus jornadas laborales. En este sentido, cabe hacer una mención a los procesos de evaluación interna del profesorado ya que en algunas universidades, especialmente en el sector privado, es el que posibilita la renovación del contrato laboral o colaboración. Estos procesos, en la mayoría de los casos, se reducen a la recopilación de las opiniones de los alumnos y no a una revisión sistemática y rigurosa de todas las funciones encomendadas al docente.

Varios trabajos de investigación - Perrenoud (2004), Cano (2005) y Zabalza (2009:79) - determinan el perfil profesional del profesorado incidiendo en la identidad y desarrollo profesional del profesor universitario; Se han concretado en las siguientes competencias: a) planificar el proceso de enseñanza-aprendizaje, b) seleccionar y presentar contenidos disciplinares, d) ofrecer informaciones y explicaciones comprensibles, e) manejar didácticamente las nuevas tecnologías, f) gestionar las metodologías de trabajo didáctico y las tareas de aprendizaje, g) relacionarse constructivamente con los alumnos, h) tutorizar a los alumnos y colegas, i) evaluar los 
aprendizajes, j) reflexionar e investigar sobre la enseñanza; por último, k) implicarse institucionalmente.

Bozu (2010:11) en un trabajo en el que analiza meticulosamente las claves y controversias de los jóvenes profesores universitarios en el contexto actual de la enseñanza universitaria, indica cuales son las principales necesidades e inquietudes de la práctica profesional del docente universitario.

Tabla 1: Principales necesidades e inquietudes sobre la práctica profesional.

Obtenido de Bozu (2010)

\begin{tabular}{|c|c|}
\hline \multicolumn{2}{|c|}{ Principales necesidades e inquietudes sobre la práctica profesional } \\
\hline \multirow{8}{*}{$\begin{array}{l}\text { El proceso } \\
\text { de formación } \\
\text { y desarrollo } \\
\text { profesional }\end{array}$} & Estabilidad profesional dentro de la universidad \\
\hline & Finalización del proceso de formación doctoral \\
\hline & $\begin{array}{l}\text { Formación permanente en el ámbito de la didáctica y la pedagogía: } \\
\text { ampliación de los conocimientos docentes }\end{array}$ \\
\hline & $\begin{array}{c}\text { Participación e integración en los grupo de innovación docente de la } \\
\text { Universidad }\end{array}$ \\
\hline & Más experiencia o práctica de la docencia universitaria \\
\hline & Trabajo colaborativo y acciones de colaboración e intercambio \\
\hline & $\begin{array}{c}\text { Establecer redes de trabajo o crear espacios formativos relacionados con } \\
\text { la práctica del aula. }\end{array}$ \\
\hline & Promover el mentoring en el ámbito de la docencia. \\
\hline \multirow{11}{*}{ La práctica docente } & Conseguir proyectos financiados de innovación docente \\
\hline & Estimular a los alumnos a hacerse preguntas inteligentes \\
\hline & Mejorar los procesos de trabajo en grupo de los estudiantes \\
\hline & Trabajar más en las técnicas de participación activa de los estudiantes \\
\hline & $\begin{array}{c}\text { Transmitir y lograr en los estudiantes el objetivo de la transferibilidad } \\
\text { en la experiencia profesional y en el mundo laboral de los conocimientos } \\
\text { adquiridos durante la carrera }\end{array}$ \\
\hline & Potenciar las reflexión de los alumnos en los trabajos autónomos \\
\hline & Implementar las recomendaciones de Bolonia en la práctica docente \\
\hline & $\begin{array}{c}\text { Mejorar y actualizar los contenidos de la materia y de los materiales } \\
\text { docentes elaborados }\end{array}$ \\
\hline & Elaborar programas y planes docentes \\
\hline & Potenciar la tutoría \\
\hline & Crear y mantener un clima de comunicación y trabajo muy motivador \\
\hline
\end{tabular}

Cerrillo e Izuzquiza enuncian algunas de las funciones que le han sido atribuidas al profesor universitario:

Ilusionados con la tarea educativa, impulsores de equipos, sensatos "optimistas pedagógicos", mediadores del saber y también de la vida, agentes de desarrollo y de cambio social, estimuladores de "perplejidad intelectual", críticos que enseñen a pensar sobre lo que se piensa y cómo y por qué se piensa, transmisores de cultura en el sentido más amplio y profundo que sea posible. (Cerrillo e Izuzquiza, 2005, p.5-6)

Tejada (2013:174) le atribuye, complementando lo anterior, "la planificación/ programación, impartición, evaluación, autorización, orientación, investigación, 
innovación, dinamización de la red, etc”. Quizás el que establece una categorización más exhaustiva de las funciones y actitudes que debe tener un profesor universitario es Palomero:

La calidad de la enseñanza universitaria necesita de un profesor reflexivo y crítico, motivado, responsable y profesionalmente satisfecho; comprometido con su triple misión educadora, docente e investigadora, capacitado para generar un buen clima de trabajo, dotado para la comunicación personal y para la gestión de recursos y grupos; potenciador, mediador, facilitador y guía de aprendizajes significativos; conocedor de las nuevas tecnologías de la información y la comunicación; con suficientes conocimientos sobre los procesos educativos generales propios de los niveles o áreas correspondientes a su particular dedicación. (Palomero, 2003, p.23)

\section{Objetivos}

A partir del marco teórico planteado muchos son los interrogantes que surgen aplicados a la situación laboral y académica del profesorado de Ciencias de la Comunicación en España. Los interrogantes se plasman en estos objetivos:

O1. Determinar las condiciones laborales (retribución, carga docente, gestión e investigación) y de promoción/carrera académica del profesorado de Ciencias de la Comunicación en España.

O2. Analizar cuáles son las categorías docentes predominantes en las Facultades de Ciencias de la Comunicación en España

O3. Descubrir si existen diferencias sustanciales entre los profesores de Ciencias de la Comunicación que ejercen su trabajo en las Universidades de titularidad pública y privada.

O4. Conocer la retribución que perciben los profesores de Ciencias de la Comunicación en cada una de las categorías académicas.

A partir de estos objetivos, pasamos a definir las hipótesis como explicaciones tentativas del fenómeno investigado, que formulamos como afirmaciones.

H1. Las condiciones laborales y de promoción del profesorado de Ciencias de la Comunicación no son las adecuadas para ejercer las funciones que le son exigidas en la búsqueda de la excelencia (docencia, gestión e investigación).

H2. Las categorías docentes predominantes en las facultades de Ciencias de la Comunicación en España son de carácter contratado (no funcionarial) y en concreto abundan aquellas que son de mayor precariedad (asociados, ayudantes doctores, titulares interinos y catedráticos interinos). 
H3. Las situaciones laborales, de promoción y del ejercicio de la labores propias del profesorado de Ciencias de la Comunicación son bastante peores en las Universidad privadas que en las de titularidad pública.

H4. Las retribuciones que perciben los profesores de Ciencias de la Comunicación en España no tienen una relación de proporcionalidad con las labores y funciones de responsabilidad que le han sido encomendadas con la aprobación del Plan Bolonia.

\section{Metodología}

Para poder demostrar las hipótesis y alcanzar los objetivos planteados, hemos confeccionado un cuestionario telemático a través de la aplicación Limesurvey que constaba de catorce preguntas cerradas en algunos casos se les instaba a que otorgasen una puntuación, en otras se les solicitaba que hicieran un escala de valoración y por último, se les pedía que, sobre una lista predeterminada de elementos, señalaran con una " $x$ " aquella respuesta que correspondiera.

Tabla 2: Ficha resumen de los parámetros de la investigación empírica

\begin{tabular}{|c|c|}
\hline Enfoque de la investigación & Empírica, exploratoria y descriptivo-analítica \\
\hline Muestra & $\begin{array}{c}\text { No probabilística, accesible, voluntaria, estratégica } \\
\text { Profesores que imparten docencia en algún grado de Ciencias } \\
\text { de la Comunicación en España }\end{array}$ \\
\hline Método & Cuantitativo \\
\hline Cuestionario & $\begin{array}{l}\text { Respuesta cerrada de selección múltiple } \\
\text { Escala de Likert }\end{array}$ \\
\hline Tamaño muestral & $\begin{array}{l}543 \text { profesores que imparten docencia en alguna materia de } \\
\text { Ciencias de la Comunicación en alguna Universidad/Facultad } \\
\text { española }\end{array}$ \\
\hline Forma & LimeSurvey \\
\hline Mecánica de seguimiento & Mail \\
\hline $\begin{array}{l}\text { Procesamiento de la } \\
\text { información }\end{array}$ & SPSS 18.0 \\
\hline Método de muestreo & Muestreo por cuotas y de conveniencia \\
\hline Fecha del trabajo de campo & Febrero 2013 \\
\hline
\end{tabular}

Los datos recogidos en cada una de las preguntas fueron codificados e introducidos en el equipo del centro de datos de la Universidad San Pablo CEU utilizando una programación propia. Para el tratamiento y explotación de los datos se ha empleado el programa SPSS v.18. 


\section{Resultados: descripción y análisis}

A continuación se lleva a cabo el análisis descriptivo de las variables del cuestionario en el que han participado un total de 445 individuos de los cuales 211 eran mujeres, lo que representa un 47,4\%; y 234 hombres, lo que supone un 52,6\%. En la siguiente tabla mostramos la participación de tramos de edad. Esos tramos fueron establecidos con arreglo al desarrollo de la carrera docente de un profesor: junior (20-35 años), senior (36-45 años), agregados (46-55 años), y catedráticos (más de 55 años). Hemos considerado las primeras etapas de docencia e investigación hasta la consolidación de las mismas.

Tabla 3. Tramos de edad de la muestra que participó en el estudio

\begin{tabular}{|c|c|c|c|}
\hline \multicolumn{2}{|c|}{ Edad } & Frecuencia & Porcentaje \\
\hline \multirow{4}{*}{ Válidos } & $20-35$ años & 87 & $19,6 \%$ \\
\cline { 2 - 4 } & $36-45$ años & 173 & $38,9 \%$ \\
\cline { 2 - 4 } & $46-55$ años & 125 & $28,1 \%$ \\
\cline { 2 - 4 } & Más de 55 años & 60 & $13,5 \%$ \\
\cline { 2 - 4 } & Total & 445 & $100,0 \%$ \\
\hline
\end{tabular}

En el estudio participaron profesores de un total de cuarenta y nueve facultades/ universidades de Ciencias de la Comunicación, de las cuales 18 son de titularidad privada y 31 pertenecientes al Estado.

Tabla 4. Universidades participantes en el estudio empírico

\begin{tabular}{|c|c|c|c|c|c|c|c|}
\hline Universidad & $\mathbf{\%}$ & Universidad & $\mathbf{\%}$ & Universidad & $\mathbf{\%}$ & Universidad & $\%$ \\
\hline $\begin{array}{c}\text { Católica San } \\
\text { Antonio }\end{array}$ & 1,6 & Murcia & 0,7 & $\begin{array}{c}\text { Europea Miguel } \\
\text { de Cervantes }\end{array}$ & 1,6 & $\begin{array}{c}\text { Abat Oliba- } \\
\text { CEU }\end{array}$ & 3,6 \\
\hline $\begin{array}{c}\text { Complutense } \\
\text { de Madrid }\end{array}$ & 13,7 & Navarra & 1,8 & $\mathrm{~F}^{\mathbf{o}}$ de Vitoria & 0,7 & $\begin{array}{c}\text { Autónoma de } \\
\text { Barcelona }\end{array}$ & 2,5 \\
\hline Burgos & 1,1 & Salamanca & 1,6 & UNIR & 0,9 & $\begin{array}{c}\text { Cardenal } \\
\text { Herrera-CEU }\end{array}$ & 2,7 \\
\hline Cádiz & 0,4 & $\begin{array}{c}\text { San Pablo- } \\
\text { CEU }\end{array}$ & 1,3 & $\begin{array}{c}\text { Miguel } \\
\text { Hernández }\end{array}$ & 0,9 & Alicante & 2,9 \\
\hline $\begin{array}{c}\text { Castilla-La } \\
\text { Mancha }\end{array}$ & 1,1 & Sevilla & 3,6 & UNED & 0,2 & Barcelona & 0,2 \\
\hline Deusto & 0,2 & Valladolid & 0,4 & $\begin{array}{c}\text { Pontificia } \\
\text { Salamanca }\end{array}$ & 1,3 & Girona & 1,1 \\
\hline Extremadura & 0,9 & Vigo & 4,0 & Rey Juan Carlos & 9,9 & Valencia & 0,4 \\
\hline Granada & 1,1 & Zaragoza & 1,3 & San Jorge & 1,6 & Vic & 0,9 \\
\hline La Laguna & 0,4 & País Vasco & 5,8 & Coruña & 1,3 & UIC & 0,4 \\
\hline Málaga & 2,9 & $\begin{array}{c}\text { Europea de } \\
\text { Madrid }\end{array}$ & 0,4 & $\begin{array}{c}\text { Santiago de } \\
\text { Compostela }\end{array}$ & 1,6 & $\begin{array}{c}\text { Jaume I } \\
\text { Castelló }\end{array}$ & 1,8 \\
\hline UOC & 0,2 & Pompeu Fabra & 3,1 & $\begin{array}{c}\text { Ramón Llul } \\
\text { (Blanquerna) }\end{array}$ & 5,4 & $\begin{array}{c}\text { Rovira i } \\
\text { Virgili }\end{array}$ & 1,3 \\
\hline $\begin{array}{c}\text { IE } \\
\text { universidad }\end{array}$ & 0,9 & UDIMA & 1,1 & Antonio Nebrija & 1,3 & $\begin{array}{c}\text { Camilo José } \\
\text { Cela }\end{array}$ & 2,7 \\
\hline
\end{tabular}




\begin{tabular}{|c|c|l|}
\hline $\begin{array}{c}\text { Carlos III de } \\
\text { Madrid }\end{array}$ & 2,5 & \\
\hline
\end{tabular}

Para hacer más operativo el estudio de los datos establecimos una tabla de contingencia de los datos anteriores en función e la titularidad de la Universidad/Facultad.

Tabla 5. Tabla de contingencia de procedencia de la universidad (pública/privada) y sexo de la muestra participante

\begin{tabular}{|c|c|c|c|c|c|}
\hline & \multicolumn{2}{|c|}{ Sexo } & \multirow{2}{*}{ Total } \\
\hline & & & Femenino & Masculino & \\
\hline \multirow{8}{*}{ Universidad } & \multirow{4}{*}{ Pública } & Recuento & 143 & 163 & 306 \\
\hline & & $\begin{array}{l}\% \text { dentro de } \\
\text { Universidad }\end{array}$ & $46,7 \%$ & $53,3 \%$ & $100,0 \%$ \\
\hline & & $\%$ dentro de Sexo & $67,8 \%$ & $69,7 \%$ & $68,8 \%$ \\
\hline & & $\%$ del total & $32,1 \%$ & $36,6 \%$ & $68,8 \%$ \\
\hline & \multirow{4}{*}{ Privada } & Recuento & 68 & 71 & 139 \\
\hline & & $\begin{array}{l}\% \text { dentro de } \\
\text { Universidad }\end{array}$ & $48,9 \%$ & $51,1 \%$ & $100,0 \%$ \\
\hline & & $\%$ dentro de Sexo & $32,2 \%$ & $30,3 \%$ & $31,2 \%$ \\
\hline & & $\%$ del total & $15,3 \%$ & $16,0 \%$ & $31,2 \%$ \\
\hline \multirow{4}{*}{\multicolumn{2}{|c|}{ Total }} & Recuento & 211 & 234 & 445 \\
\hline & & $\begin{array}{l}\% \text { dentro de } \\
\text { Universidad }\end{array}$ & $47,4 \%$ & $52,6 \%$ & $100,0 \%$ \\
\hline & & $\%$ dentro de Sexo & $100,0 \%$ & $100,0 \%$ & $100,0 \%$ \\
\hline & & $\%$ del total & $47,4 \%$ & $52,6 \%$ & $100,0 \%$ \\
\hline
\end{tabular}

En la (tabla 5) se puede apreciar una mayor participación de profesores de la universidad pública $68,8 \%$ que de los profesores que imparten docencia e investigación en la privada $31,2 \%$. Además podemos afirmar, que existe un equilibrio por género en los profesores que imparten docencia en esta disciplina científica tanto en la Universidad pública como en la privada.

Veamos en la siguiente tabla en qué categoría de contrato están ubicados los profesores que han participado en la muestra.

Tabla 6. Categoría docente de los profesores que han participado en el estudio

\begin{tabular}{|c|c|c|c|}
\hline \multicolumn{2}{|c|}{ Categoría docente/académica } & Frecuencia & Porcentaje \\
\hline \multirow{4}{*}{} & Asociado t. parcial & 113 & $25,4 \%$ \\
\cline { 2 - 4 } & Asociado t. completo & 44 & $9,9 \%$ \\
\cline { 2 - 4 } & Ayudante doctor & 44 & $9,9 \%$ \\
\cline { 2 - 4 } & Contratado doctor & 103 & $23,1 \%$ \\
\cline { 2 - 4 } & Titular interino & 29 & $6,5 \%$ \\
\cline { 2 - 4 } & Catedrático interino & 4 & $0,9 \%$ \\
\cline { 2 - 4 } & Titular universidad & 89 & $20,0 \%$ \\
\cline { 2 - 4 } & Catedrático universidad & 19 & $4,3 \%$ \\
\cline { 2 - 4 } & Total & 445 & $100,0 \%$ \\
\hline
\end{tabular}


Observamos una gran precariedad en la situación de inestabilidad del profesorado de Ciencias de la Comunicación ya que tan sólo el 24,3 \% de la muestra está en una situación estable (funcionarios). El resto, lo que supone el $74.7 \%$ está en una situación de incertidumbre; porque incluso los profesores contratados doctores, que suponen el 23,1\% son contratados laborales que pueden ser despedidos en cualquier momento por la Administración Pública. Los ayudantes doctores $(9,9 \%)$ deben conseguir la acreditación a titular antes de cinco años para que la Universidad les pueda consolidar. La Ley Orgánica 4/2007, de 12 de abril, por la que se modifica la Ley Orgánica 6/2001, de 21 de diciembre, de Universidades en el artículo 50 apartado d) refleja claramente el carácter temporal de la permanencia de un profesor en esta figura.

La duración del contrato no podrá ser inferior a un año ni superior a cinco, pudiendo prorrogarse o renovarse si se hubiera concertado por duración inferior a la máxima, siempre que la duración total no exceda de los indicados cinco años. En cualquier caso, el tiempo total de duración conjunta entre esta figura contractual y la prevista en el artículo anterior, en la misma o distinta universidad, no podrá exceder de ocho años. Las situaciones de incapacidad temporal, maternidad y adopción o acogimiento durante el período de duración del contrato, interrumpirán su cómputo. (Ley Orgánica 6/2001, art.50,aprtdo.d)

En el caso de no obtener la acreditación a la figura de profesor titular en el período fijado, el profesor puede ser despedido. El resto de figuras (asociados a tiempo parcial, completo, titular interino y catedrático interino) que suman un porcentaje del $42,7 \%$ son profesores con salarios muy precarios tal y como observaremos en tablas posteriores y que además cada año se revisa por parte de los órganos de gobiernos de las Universidades la continuidad de sus contratos.

Sorprende observar la gran cantidad de profesores que participan en la muestra y que se encuentran en situación de precariedad e inestabilidad. Así, las figuras de asociados, ayudantes doctores, titulares interinos y catedráticos interinos son figuras que interesan a los resultados económicos de la Universidades porque suponen la más alta cualificación al menor coste pero habría que hacer un estudio más pormenorizado sobre cómo puede afectar eso a la calidad y excelencia que persiguen las facultades/universidades. En esta tabla a través de las celdas de color rojo vemos algunas disfunciones. Por ejemplo es cuando menos llamativo, ver cómo siete individuos de la muestra con más de 46 años de edad se encuentran aún en figuras que están pensadas para profesores más jóvenes. O profesores contratados doctores con más de 55 años cuando en ese tramo de edad lo más lógico dentro de su carrera docente ya fuesen profesores titulares de universidad u optando a cátedra. . Por último mencionar los 14 profesores (titulares y catedráticos) que están con carácter interino con más de 46 años de edad. Nos podemos hacer una idea de la inestabilidad laboral de este colectivo al que además se le exige exclusividad e incompatibilidad con cualquier otro tipo de trabajo retribuido. 
Tabla 7. Tabla de contingencia tipo de contrato y edad

\begin{tabular}{|c|c|c|c|c|c|c|}
\hline & \multicolumn{4}{|c|}{ Edad } & \multirow[b]{2}{*}{ Total } \\
\hline & & 20-35 años & $\begin{array}{c}36-45 \\
\text { años }\end{array}$ & $\begin{array}{c}46-55 \\
\text { años }\end{array}$ & $\begin{array}{l}\text { Más de } 55 \\
\text { años }\end{array}$ & \\
\hline \multirow{8}{*}{ Contrato } & $\begin{array}{l}\text { Asociado t. } \\
\text { parcial }\end{array}$ & 27 & 35 & 38 & 13 & 113 \\
\hline & $\begin{array}{l}\text { Asociado t. } \\
\text { completo }\end{array}$ & 14 & 13 & 14 & 3 & 44 \\
\hline & $\begin{array}{l}\text { Ayudante } \\
\text { doctor }\end{array}$ & 19 & 17 & 7 & 1 & 44 \\
\hline & $\begin{array}{c}\text { Contratado } \\
\text { doctor }\end{array}$ & 19 & 59 & 20 & 5 & 103 \\
\hline & $\begin{array}{c}\text { Titular } \\
\text { interino }\end{array}$ & 5 & 14 & 7 & 3 & 29 \\
\hline & $\begin{array}{c}\text { Catedrático } \\
\text { interino }\end{array}$ & 0 & 0 & 2 & 2 & 4 \\
\hline & $\begin{array}{c}\text { Titular } \\
\text { universidad }\end{array}$ & 3 & 34 & 34 & 18 & 89 \\
\hline & $\begin{array}{l}\text { Catedrático } \\
\text { universidad }\end{array}$ & 0 & 1 & 3 & 15 & 19 \\
\hline \multicolumn{2}{|c|}{ Total } & 87 & 173 & 125 & 60 & 445 \\
\hline
\end{tabular}

Esta tabla complementa a la anterior y podemos ver la media de edad para cada una de las categorías académicas. Vemos cómo la media del profesorado total de Ciencias de la Comunicación se puede situar en 44 años. No obstante es singular la media de edad para figuras, como la de ayudante doctor, que debiere ser en principio para jóvenes que acaban de finalizar la tesis doctoral y que, sin embargo, está situada en 38 años. Resulta desalentador comprobar cómo no se consigue una estabilidad funcionarial (entrar en el cuerpo de funcionarios titulares) hasta las 48 años de edad.

Tabla 8. Edad media de cada una de las categorías académicas de los profesores de Ciencias de la Comunicación

\begin{tabular}{|c|c|c|c|c|}
\hline Contrato & N & Media & Mediana & Desv. típ. \\
\hline Asociado t. parcial & 113 & 43,7611 & 43,0000 & 8,99035 \\
\hline Asociado t. completo & 44 & 41,7955 & 40,5000 & 9,25750 \\
\hline Ayudante doctor & 44 & 38,5227 & 37,0000 & 7,50345 \\
\hline Contratado doctor & 103 & 42,3010 & 42,0000 & 6,91947 \\
\hline Titular interino & 29 & 43,0000 & 42,0000 & 8,21584 \\
\hline Catedrático interino & 4 & 57,5000 & 59,0000 & 6,80686 \\
\hline Titular universidad & 89 & 48,2809 & 48,0000 & 7,85087 \\
\hline $\begin{array}{c}\text { Catedrático } \\
\text { universidad }\end{array}$ & 19 & 58,3158 & 59,0000 & 6,64206 \\
\hline Total & 445 & 44,3101 & 43,0000 & 9,05031 \\
\hline
\end{tabular}

Veamos en el siguiente gráfico el número de créditos que imparten los profesores de Ciencias de la comunicación en primer y segundo semestre incluyendo docencia en grado, master y doctorado. 
Tabla 9. Número de créditos medios que asumen los profesores de Ciencias de la Comunicación (incluyendo los créditos de Licenciatura/Grado, Máster y Doctorado)

\begin{tabular}{|c|c|c|c|}
\hline & $\begin{array}{c}\text { Número de } \\
\text { créditos 1 } \\
\text { Semestre }\end{array}$ & $\begin{array}{c}\text { Número de } \\
\text { créditos 2 } \\
\text { Semestre }\end{array}$ & $\begin{array}{c}\text { Número de } \\
\text { créditos anuales }\end{array}$ \\
\hline $\mathrm{N}$ & 291 & 279 & 267 \\
\hline Media & 11,69 & 10,08 & 22,20 \\
\hline Mediana & 12,00 & 9,00 & 22,00 \\
\hline Desv. típ. & 7,848 & 7,279 & 10,274 \\
\hline Error típ. de la media &, 460 &, 436 &, 629 \\
\hline
\end{tabular}

Podemos observar en esta tabla resumen que el número de créditos medios que un profesor de Ciencias de la Comunicación imparte por semestre está alrededor de 11,5 con lo que por curso académico se sitúa en 22 créditos aproximadamente. En un análisis pormenorizado de los casos, hemos podido observar como esa tendencia varía en función de la titularidad de la universidad. De este modo podemos afirmar, generalizando, que los profesores de universidad privada soportan una mayor carga de créditos por semestre, situándose como media anual en 30 créditos.

Además de las labores propias de docencia queríamos conocer si los profesores tenían dentro de sus facultades/universidades alumnos asignados dentro de un plan específico de Acción Tutorial.

Tabla 10. Asignación de alumnos para ejercer la labor de acción tutorial

\begin{tabular}{|c|c|c|c|}
\hline \multicolumn{2}{|c|}{} & Frecuencia & Porcentaje \\
\hline \multirow{3}{*}{ Válidos } & Sí & 188 & 42,2 \\
\cline { 2 - 4 } & No & 136 & 30,6 \\
\cline { 2 - 4 } & Total & 324 & 72,8 \\
\hline Perdidos & Sistema & 121 & 27,2 \\
\hline \multicolumn{2}{|c|}{ Total } & 445 & 100,0 \\
\hline
\end{tabular}

Observamos que el $42,2 \%$ posee alumnos asignados desde el inicio de curso mientras que un 30,6\% no poseen la labor de torización específica dentro de un Plan de Acción Tutorial. Un 27,2\% no contestaron esta pregunta. Al aplicar el estadístico de Levene, entre plan de acción tutorial y tipo de universidad/facultad obtuvimos una significación de ,002 con lo que podemos afirmar que el plan de acción tutorial está más implantado en las universidad de titularidad privada. Con lo que los profesores de universidad privada asumen desde el inicio de curso un número de alumnos para ejercer con ellos una tutoría académica y/o personal. Es precisamente el seguimiento personalizado y continuado del alumno, uno de los principales valores añadidos de la universidad privada.

En cuanto a la participación en proyectos de investigación competitiva observamos que el 45,6\% de los encuestados está realizando tareas de investigación dentro de un grupo de investigación competitiva. Un $27,2 \%$ de los mismos afirma no parti- 
cipar en ningún proyecto de investigación. Esta notable participación se debe a la alta puntuación que otorgan las agencias de evaluación del profesorado para obtener la evaluación positiva en cualquiera de las categorías a las que se puede postular un profesor.

Tabla 11. Participación en algún proyecto de investigación competitiva de carácter regional, autonómico, nacional o internacional

\begin{tabular}{|c|c|c|c|}
\hline & & Frecuencia & Porcentaje \\
\hline \multirow{3}{*}{ Válidos } & Sí & 203 & 45,6 \\
\hline & No & 121 & 27,2 \\
\hline & Total & 324 & 72,8 \\
\hline Perdidos & Sistema & 121 & 27,2 \\
\hline \multicolumn{2}{|c|}{ Total } & 445 & 100,0 \\
\hline
\end{tabular}

Al aplicar el la prueba de chi-cuadrado, entre la participación en proyectos de investigación competitiva y tipo de universidad/facultad (pública/privada) obtuvimos una significación de ,011. Lo que quiere decir que existe una diferencia significativa, entre los profesores que imparten docencia e investigación en las facultades/universidad públicas y los de la privada; siendo la participación en proyectos de investigación muy superior por parte de los profesores de la Universidad pública.

Tabla 12. Tabla de contingencia entre la titularidad de la universidad y la participación en proyectos de investigación

\begin{tabular}{|c|c|c|c|c|c|}
\hline \multicolumn{2}{|c|}{} & $\begin{array}{c}\text { iParticipa en algún proyecto } \\
\text { de investigación competitiva } \\
\text { de carácter regional, } \\
\text { autonómico, nacional o } \\
\text { internacional? }\end{array}$ & \multirow{2}{*}{ Total } \\
\cline { 3 - 6 } & & \multicolumn{1}{|c|}{ Sí } & No & \\
\hline \multirow{3}{*}{ Universidad } & \multirow{2}{*}{ Pública } & Recuento & 150 & 76 & 226 \\
\cline { 3 - 6 } & \multirow{3}{*}{$\begin{array}{c}\text { \% dentro de } \\
\text { Universidad }\end{array}$} & $66,4 \%$ & $33,6 \%$ & $100,0 \%$ \\
\cline { 3 - 6 } & \multirow{3}{*}{ Privada } & Recuento & 53 & 45 & 98 \\
\cline { 3 - 6 } & $\begin{array}{c}\text { \% dentro de } \\
\text { Universidad }\end{array}$ & $54,1 \%$ & $45,9 \%$ & $100,0 \%$ \\
\hline \multirow{2}{*}{ Total } & Recuento & 203 & 121 & 324 \\
\cline { 3 - 6 } & $\begin{array}{c}\% \text { dentro de } \\
\text { Universidad }\end{array}$ & $62,7 \%$ & $37,3 \%$ & $100,0 \%$ \\
\hline
\end{tabular}

La capacidad investigadora (productividad científica) de los profesores es medida por la CNEAI (Centro Nacional de la Evaluación de la Actividad Investigadora) que valida la producción científica por tramos de seis años. Veamos en la siguiente tabla la capacidad de producción científica de los profesores de Ciencias de la Comunicación en España. 
Tabla 13. Número de sexenios que posee en su haber

\begin{tabular}{|c|c|c|c|}
\hline \multicolumn{2}{|c|}{ Número de sexenios } & Frecuencia & Porcentaje \\
\hline \multirow{4}{*}{ Válidos } & 0 & 238 & 53,5 \\
\cline { 2 - 4 } & 1 & 51 & 11,5 \\
\cline { 2 - 4 } & 2 & 13 & 2,9 \\
\cline { 2 - 4 } & 3 & 4 &, 9 \\
\cline { 2 - 4 } & 4 & 4 &, 9 \\
\cline { 2 - 4 } & 5 & 2 &, 4 \\
\cline { 2 - 4 } & Total & 312 & 70,1 \\
\hline Perdidos & Sistema & 133 & 29,9 \\
\hline \multicolumn{2}{|c|}{ Total } & 445 & 100,0 \\
\hline
\end{tabular}

El $16,6 \%$ de los casos afirma poseer en su haber un sexenio de investigación reconocido por el organismo de evaluación. Mientras una mayoría del 53,5\% de los encuestados manifiesta no poseer un tramo de investigación reconocido. Esta cifra, que a priori puede sorprender en negativo, se puede deber fundamentalmente a tres causas: en la actualidad existen escasas revistas de Comunicación indexadas en índices de calidad de reconocido prestigio como ISI y SCOPUS, por otro lado en la Universidad pública tan sólo pueden solicitar la evaluación Catedráticos, Titulares y profesores Contratados Doctores (el resto de figuras no tienen dicha posibilidad, ni siquiera los titulares o catedráticos interinos), y por último, muchas universidades privadas no tienen convenio de colaboración con la CNEAI con lo que sus profesores no pueden solicitar ser evaluados. Todo ello redunda en la escasa posibilidad que poseen los profesores de obtener evaluación positiva de sexenio; y por ende, de obtener acreditaciones para optar a los cuerpos de funcionarios y poder liderar como investigadores principales proyectos de investigación competitivos. Analicemos en la siguiente tabla, el número de sexenios que poseen en su haber las personas que han afirmado poseer un sexenio de investigación. De ellos el 11,5\% posee un tramo, el $2,9 \%$ posee dos tramos y el resto de tramos no llega al $1 \%$.

Tabla 14. Tabla de contingencia entre la titularidad de la universidad y el número de sexenios de investigación

\begin{tabular}{|c|c|c|c|c|c|c|c|c|c|}
\hline & \multicolumn{6}{|c|}{$\begin{array}{l}\text { Indique el número de sexenios que posee en } \\
\text { su haber }\end{array}$} & \multirow{2}{*}{ Total } \\
\hline & & & 0 & 1 & 2 & 3 & 4 & 5 & \\
\hline \multirow{4}{*}{ Universidad } & \multirow[b]{2}{*}{ Pública } & Recuento & 152 & 44 & 12 & 4 & 4 & 2 & 218 \\
\hline & & $\begin{array}{l}\text { \% dentro de } \\
\text { Universidad }\end{array}$ & $69,7 \%$ & $20,2 \%$ & $5,5 \%$ & $1,8 \%$ & $1,8 \%$ &, $9 \%$ & $100,0 \%$ \\
\hline & \multirow[b]{2}{*}{ Privada } & Recuento & 86 & 7 & 1 & 0 & 0 & 0 & 94 \\
\hline & & $\begin{array}{l}\% \text { dentro de } \\
\text { Universidad }\end{array}$ & $91,5 \%$ & $7,4 \%$ & $1,1 \%$ &, $0 \%$ &, $0 \%$ &, $0 \%$ & $100,0 \%$ \\
\hline \multirow{2}{*}{\multicolumn{2}{|c|}{ Total }} & Recuento & 238 & 51 & 13 & 4 & 4 & 2 & 312 \\
\hline & & $\begin{array}{l}\text { \% dentro de } \\
\text { Universidad }\end{array}$ & $76,3 \%$ & $16,3 \%$ & $4,2 \%$ & $1,3 \%$ & $1,3 \%$ & ,6\% & $100,0 \%$ \\
\hline
\end{tabular}


Al aplicar el la prueba de chi-cuadrado, entre la posesión de sexenio de investigación y tipo de universidad/facultad (pública/privada) obtuvimos una significación de ,001. Este dato nos lleva a afirmar que el mayor número de sexenios puede venir determinado por la titularidad de la universidad en la que se encuentra el profesor desarrollando sus funciones.

En cuanto al número de tesis dirigidas, podemos observar cómo el 42,2\% de la muestra no ha dirigido ninguna tesis a pesar de ser una de las funciones enmarcadas en la faceta investigadora más importantes, teóricamente, para el profesor universitario. El 22,9 \% ha dirigido entre 1 y 4 tesis doctorales. Un $4,3 \%$ ha dirigido entre 5 y 10 ; y son pocos los profesores de Ciencias de la Comunicación, que han dirigido más de 10 tesis. Curiosamente los que más tesis dirigen están ubicados en la categoría de profesores Contratados doctores que son la figura que más méritos está dispuesto hacer pasa saltar a las categorías de funcionario. En segundo lugar estarían los ayudantes doctores, que al igual que los anteriores están en una fase de consecución de méritos para conseguir la estabilización de la figura hacia titulares. Como bien refleja Ley Orgánica 4/2007el ayudante doctor tiene un plazo de cinco años para conseguir la acreditación de titular de universidad. Por el contrario los profesores Catedráticos y titulares son los que en proporción menos tesis doctorales dirigen.

Tabla 15. Tabla de frecuencia respecto al número de tesis doctoral que han dirigido o co-dirigido

\begin{tabular}{|c|c|c|c|}
\hline & & Frecuencia & Porcentaje \\
\hline \multirow{5}{*}{ Válidos } & 0 & 188 & 42,2 \\
\hline & $1-4$ & 102 & 22,9 \\
\hline & $5-10$ & 19 & 4,3 \\
\hline & $>10$ & 6 & 1,3 \\
\hline & Total & 315 & 70,8 \\
\hline Perdidos & Sistema & 130 & 29,2 \\
\hline \multicolumn{2}{|c|}{ Total } & 445 & 100,0 \\
\hline
\end{tabular}

Con la introducción del Trabajo Fin de Grado (TFG) en los planes de estudios como materia obligatoria4 se ha incrementado la cantidad de profesores que dirigen TFG. Así lo refleja la siguiente tabla. El 30,8\% de los profesores que han participado en la muestra han dirigido de 1 a 10 trabajos; el 3,6\% ha tutelado entre 11 y 15 trabajos; y por último, el 3,4\% ha dirigido más de 15 trabajos fin de grado. Esta cifra irá in-crescendo a lo largo de los años ya que tan sólo han llegado, en el mejor de los casos, 2 promociones desde que se implantara en las enseñanzas universitarias en Plan Bolonia. 
Tabla 16. Tabla de frecuencia respecto al número de TFG que han dirigido o co-dirigido

\begin{tabular}{|c|c|c|c|}
\hline \multicolumn{2}{|c|}{ Número de TFG's } & Frecuencia & Porcentaje \\
\hline \multirow{4}{*}{ Válidos } & 0 & 149 & 33,5 \\
\cline { 2 - 4 } & $1-10$ & 137 & 30,8 \\
\cline { 2 - 4 } & $11-15$ & 16 & 3,6 \\
\cline { 2 - 4 } & $>15$ & 15 & 3,4 \\
\cline { 2 - 4 } & Total & 317 & 71,2 \\
\hline Perdidos & Sistema & 128 & 28,8 \\
\hline \multicolumn{2}{|c|}{ Total } & 445 & 100,0 \\
\hline
\end{tabular}

Una de las variables más importantes para identificar un sistema educativo excelente y de calidad es la retribución que percibe el profesorado para las tareas de docencia, gestión e investigación que le han sido encomendadas. En la siguiente tabla mostramos la radiografía de la retribución por categoría profesional de los profesores de Ciencias de la Comunicación en España.

Tabla 17. Tabla de frecuencia respecto a la retribución salarial que percibe el profesorado

\begin{tabular}{|c|c|c|c|c|}
\hline \multicolumn{2}{|c|}{ Tramos de retribución } & \multirow{2}{*}{$\begin{array}{c}\text { Frecuencia } \\
56 \\
\end{array}$} & \multirow{2}{*}{$\begin{array}{c}\text { Porcentaje } \\
12,6 \% \\
\end{array}$} & \multirow{2}{*}{$\begin{array}{c}\% \\
\text { Global } \\
\text { por } \\
\text { Tramo }\end{array}$} \\
\hline \multirow{3}{*}{$\begin{array}{c}\text { Tramo } 1 \\
\text { Bajo } \\
\text { (ASOCIADOS } \\
\text { TITULARES INT. } \\
\text { CATEDRÁTICOS } \\
\text { INT.) } \\
\end{array}$} & Menos de 10.000 euros & & & \\
\hline & De 10.000 euros a 13.000 euros & 16 & $3,6 \%$ & \multirow{2}{*}{$17,50 \%$} \\
\hline & De 13.001 euros a 16.000 euros & 6 & $1,3 \%$ & \\
\hline \multirow{5}{*}{$\begin{array}{c}\text { Tramo } 2 \\
\text { Bajo- Medio } \\
\text { (AYUDANTE } \\
\text { DOCTOR) }\end{array}$} & De 16.001 euros a 19.000 euros & 15 & $3,4 \%$ & \multirow{5}{*}{$21,10 \%$} \\
\hline & De 19.001 euros a 22.000 euros & 19 & $4,3 \%$ & \\
\hline & De 22.001 euros a 25.000 euros & 18 & $4,0 \%$ & \\
\hline & De 25.001 euros a 28.000 euros & 14 & $3,1 \%$ & \\
\hline & De 28.001 euros a 31.000 euros & 28 & $6,3 \%$ & \\
\hline \multirow{7}{*}{$\begin{array}{c}\text { Tramo } 3 \\
\text { Normal } \\
\text { (CONTRATADO } \\
\text { DOCTOR) }\end{array}$} & De 31.001 euros a 34.000 euros & 20 & $4,5 \%$ & \multirow{7}{*}{$23,10 \%$} \\
\hline & De 34.001 euros a 37.000 euros & 21 & $4,7 \%$ & \\
\hline & De 37.001 euros a 40.000 euros & 18 & $4,0 \%$ & \\
\hline & De 40.001 euros a 43.000 euros & 14 & $3,1 \%$ & \\
\hline & De 43.001 euros a 46.000 euros & 11 & $2,5 \%$ & \\
\hline & De 46.001 euros a 49.000 euros & 11 & $2,5 \%$ & \\
\hline & De 49.001 euros a 52.000 euros & 8 & $1,8 \%$ & \\
\hline \multirow{5}{*}{$\begin{array}{c}\text { Tramo } 4 \\
\text { Alto } \\
\text { (FUNCIONARIOS } \\
\text { TITULARES } \\
\text { CATEDRÁTICOS) }\end{array}$} & De 52.001 euros a 55.000 euros & 5 & $1,1 \%$ & \multirow{5}{*}{$4,40 \%$} \\
\hline & De 55.001 euros a 58.000 euros & 4 & $0,9 \%$ & \\
\hline & De 58.001 euros a 61.000 euros & 2 & $0,4 \%$ & \\
\hline & De 61.001 euros a 64.000 euros & 7 & $1,6 \%$ & \\
\hline & Más de 65.000 euros & 2 & $0,4 \%$ & \\
\hline
\end{tabular}




\begin{tabular}{|c|cc|c|c|c|}
\hline & Total & 295 & $66,3 \%$ & $66,3 \%$ \\
\hline Perdidos & Sistema & 150 & $33,7 \%$ & $33,7 \%$ \\
\hline \multicolumn{2}{|c|}{ Total } & 445 & 100,0 & \\
\hline
\end{tabular}

La (tabla 17) muestra las retribuciones recodificadas en 4 tramos de retribución salarial. En el primer tramo están ubicados los profesores asociados a tiempo completo y parcial, titulares interinos y catedráticos interinos. En la segunda franja están ubicados los profesores ayudantes doctores. En la tercera franja los profesores contratados doctores y en la última franja los profesores funcionarios titulares y catedráticos. Como observamos son muy pocos los profesores que están ubicados en el tramo salarial alto. Sin embargo el 38,60\% de la muestra se halla en una situación retributiva baja $(17,50 \%)$ y baja media $(21,10 \%)$.

\section{Conclusiones}

A partir de los resultados obtenidos establecemos las conclusiones más destacadas de este estudio empírico. Además, nos parece oportuno sintetizar las ideas principales desarrolladas en el marco teórico y derivadas de la revisión bibliográfica.

Los textos jurídicos de la transposición de la Declaración de Bolonia han hecho mucho énfasis en aspectos relacionados con el estudiante y su proceso de aprendizaje. Por el contrario, se han omitido aspectos esenciales de la figura del profesor, al que cada vez se le encomiendan un mayor número de funciones, tal y como hemos podido ver en este capitulo, con una mayor precariedad de recursos económicos y humanos. Esta reforma de la enseñanza superior se ha querido hacer en España, a nuestro juicio, de forma precipitada con un coste ínfimo y sin contar con las infraestructuras adecuadas para poder desarrollar adecuadamente las metodologías docentes y actividades de enseñanza-aprendizaje que se requieren en el modelo propuesto por el Espacio Europeo de Educación Superior,

Además, la coyuntura económica global afecta, por supuesto, al sistema de financiación de las Universidades, que no disponen de los recursos suficientes para poder aumentar el número de plazas docentes ni para consolidar a aquellos profesores que han conseguido acreditarse para categorías académicas superiores de las que se encuentran actualmente (con la consiguiente frustración del profesional). Ante la imposibilidad de convocar nuevas plazas en el sector público o aumentar el número de contratados en el privado y las fuertes restricciones económicas, es el profesor de nuevo quien tiene que soportar el peso de esta reforma, viéndose afectado fundamentalmente por un incremento en el número de créditos que tiene que impartir y una ralentización de la carrera de promoción docente que tiene como consecuencia un nivel salarial reducido. Veíamos en la (tabla 7) la disfunción y "tapón" que se está produciendo en la carrera docente del profesorado. Observábamos algunos casos llamativos de profesores con edad de más de 46 años se encontraban en categorías laborales (por ejemplo ayudante doctor) pensadas para el inicio de la carrera docente 
de un profesor universitario. En el caso de las universidades privadas esta situación se agudiza más aún por el descenso generalizado del número de matrículas, fuente principal de ingresos de este tipo de centros.

Estas circunstancias reducen las horas destinadas a la investigación que, sin embargo, es necesaria para conseguir la acreditación del profesorado y cierta estabilidad laboral. La falta de investigación tiene como consecuencia una reducción en la calidad de la docencia impartida y la necesidad de realizar un sobreesfuerzo por parte del docente que, en muchas ocasiones, ha de financiar sus propios proyectos de investigación a pesar de ser las instituciones universitarias y su prestigio las principales beneficiadas.

Otro aspecto que denota la grave situación que atraviesa la Universidad Española en general, y las facultades de Ciencias de la Comunicación en particular, es la radiografía de las categorías docentes de los profesores que actualmente están desarrollando su labor. Predominan los contratos laborales en un porcentaje demasiado elevado sobre los contratos de funcionarios. Pero además en los primeros, las categorías que más abundan son los asociados ; que es sin duda, la figura más vulnerable y menos estable ya que, cada cierto periodo de tiempo (normalmente cada año), son renovados o revocados. También llama poderosamente la atención el número de interinos (titulares y catedráticos) que existen impartiendo docencia en los estudios de Ciencias de la Comunicación. Parece evidente que la universidad española está apostando por la renovación hacia la excelencia desde una perspectiva cuando menos discutible y necesitada de una amplia y profunda reflexión conjunta. Esto se refleja perfectamente en la (tabla 17) donde podemos ver salarios por debajo de los 16.000 euros que corresponden a las categorías laborales de asociados e interinos.

Si el panorama resulta desalentador, es en el caso de algunas facultades/universidades de titularidad privada, salvo honrosas excepciones donde los profesores se ven abocados a asumir cargas docentes por encima de los 30 créditos anuales, cargas de gestión académica referidas al seguimiento personalizado del alumno y funciones administrativas y sin prácticamente tiempo dedicado a la investigación. La labor docente en la Universidad, a pesar de exigir un alto nivel de cualificación, es retribuida con salarios ínfimos en algunos casos y condiciones precarias en un buen número de centros.

En un futuro muy cercano, todas las facultades privadas y públicas que imparten grados de Ciencias de la Comunicación tendrán que someterse al proceso de acreditación de títulos. El veredicto que realice la Agencia de Calidad al respecto de aspectos como los porcentajes mínimos de profesores acreditados (lo que refleja de modo concreto la apuesta por la investigación) y del cumplimiento de las ratio profesor/ alumno, serán definitivos para alcanzar la coherencia necesaria en la redefinición del sistema universitario español. El avance hacia la verdadera excelencia habrá de basarse en el compromiso real de todos los agentes implicados, con una definición clara y proporcionada de funciones y políticas laborales y salariales en lo relativo al profesorado, sin cuya aportación el cambio puede quedarse reducido a un procedi- 
miento burocrático continuado que pierde de vista, y a pesar del discurso teórico, a su verdadero protagonista: el alumno.

\section{Bibliografía}

BOZU, Z. (2010). "Los jóvenes profesores universitarios en el contexto actual de la enseñanza universitaria. Claves y controversias". En: Revista Iberoamericana de Educación, 51(3)

CANO, E, (2005). Cómo mejorar las competencias docentes. Guía para la autoevaluación y desarrollo de las competencias del profesorado. Barcelona. Graó.

CERRILLO MARTÍN, M. R.; IZUZQUIZA GASSET, D. (2005). Perfil del profesorado universitario. Revista electrónica interuniversitaria de formación del profesorado, 20(8-5), 1-5.

COMISIÓN de las Comunidades Europeas (2003). El papel de la Universidad en la Europa del Conocimiento, Comunicación de la comisión. Com (2003) 58 final, Bruselas. Recuperado [01/11/2013] http://www.crue.org/export/sites/Crue/procbolonia/documentos/antecedentes/7._El_papel_de_las_universidades.pdf

DECLARACIÓN DE BERGEN. Recuperado [0ㅍ1/1/2013]. http://www.bologna-bergen2005.no/Docs/00-Main_doc/050520_Bergen_Communique.pdf

DECLARACIÓN DE BERLÍN. Recuperado [03/11/2013]. http://www.bologna-bergen2005.no/Docs/00-Main_doc/030919_Berlin_Communique.PDF

DECLARACIÓN DE BOLOÑIA. Recuperado [0 $\overline{3} / 11 / 2013]$. http://europe.eu.int/ comm/education/policies/educ/bologn a/bologna.pdf

DECLARACIÓN DE PRAGA. Recuperado [03/11/2013]. http://www.bologna-bergen2005.no/Docs/00-Main_doc/010519PRAGUE_COMMUNIQUE.PDF

ESCRIBANO SOTOS, F. y PARDO GARCÍA, I. (2005). "La función del profesor universitario de economía ¿docente o investigador? Notas para un debate". En: Estudios de Economía aplicada. Vol.23-3 pags. 685-707.

FEIXAS, M. (2004). "La influencia de factores personales, institucionales y contextuales en la trayectoria y el desarrollo docente de los profesores universitarios". En: Educar, no 33, pp. 31-59.

FERREIRA, C. (2010). "Cómo se gesta un profesor universitario". En: Crítica n ${ }^{\circ}$ 969. Septiembre-Octubre 2010.

KNIGHT, P. (2005). El profesorado de Educación Superior. Formación para la excelencia. Madrid: Narcea.

LEY ORGÁNICA 4/2007, de 12 de abril, por la que se modifica la Ley Orgánica 6/2001 de 21 de diciembre de Universidades ( BOE 13-04-2007). Recuperado [05/11/2013). http://www.boe.es/boe/dias/2007/04/13/pdfs/A16241-16260.pdf

MANJÓN MURILLO, J. (2000). "Algunas funciones del profesorado universitario para el siglo XXI: consideraciones éticas”. En: Revista Fuentes Vol.2 pp.53

MARTÍNEZ-OTERO PÉREZ, V. (2010). "La voz del profesorado universitario contratado". En: Vivat Academia. no 110. Marzo. 2010. Páginas 1-32. Recuperado [08/11/2013]. http://www.ucm.es/info/vivataca/anteriores/n110/DATOSS110.htm 
MURRAY, H.G. (1193). "Summative evaluation and Faculty Development:a Synergistic Relationship?" En Weimer,M. (ed). Faculty as teachers. National Center on Postsecondary Teaching, Learning ans Assesment. The Pennsylvania State University. Pp.85-88.

PERRENAUD, PH. (2004). 10 nuevas competencias para enseñar: invitación al viaje. Barcelona: Graó.

REAL DECRETO 1393/2007, de 29 de octubre, por el que se establece la ordenación de las enseñanzas universitarias oficiales. Recuperado [08/11/2013]. http://www. boe.es/boe/dias/2007/10/30/pdfs/A44037-44048.pdf

RODRÍGUEZ ESPINAR, S. (1994). "El desarrollo profesional del profesor universitario: algunas consideraciones sobre una experiencia". En: Revista de Enseñanza Universitaria, 7/8. Instituto de Ciencias de la Educación. Universidad de Sevilla.

RODRÍGUEZ ROJO, M. (2000). "Sociedad, universidad y profesorado". En: Revista Interuniversitaria de formación del profesorado, 38, pp. 79-99.

SALAS VELASCO, M. (2010). "Financiación y crítica de la Universidad española actual" en Crítica n $^{\circ}$ 969. Septiembre-Octubre 2010.

TEJADA, J. (2013). "Profesionalización docente en la universidad: implicaciones desde la formación". En: RUSC, 10 (1), 170-184.

TORREGO, L. (2004). "Ser profesor universitario, ¿un reto en el contexto de convergencia europea?”. En: Revista Interuniversitaria de Formación de Profesorado, 18, pp. 259-268.

ZABALZA BERAZA, M. A. (2009). "Ser profesor universitario hoy". La Cuestión Universitaria. $N^{\circ}$ 5.pp.69-81.

\section{Notas}

1 Para profundizar en el proceso de armonización de la educación universitaria europea (EEES) recomendamos la lectura de las siguientes declaraciones europeas suscritas en los diferentes encuentros de la Universidad de la Sorbona (1998), Bolonia (1999), Lisboa (2000), Praga (2001), Barcelona (2002) Graz (2003), Berlín (2003), Glasgow (2005), Bergen (2005).

2 El crédito ECTS no mide solamente las horas de clase presencial del profesor sino que es una unidad de valoración del volumen total del trabajo (individual y presencial) del alumno. El profesor, además de las horas de clase, tendrá que organizar, supervisar y orientar el trabajo de los alumnos.

3 Asociado, Ayudante, Ayudante Doctor, Contratado Doctor y Profesor Visitante) y funcionario (Titulares y Catedráticos).

4 Así lo especifica el Real Decreto 1393/2007, de 29 de octubre, por el que se establece la ordenación de las enseñanzas universitarias oficiales. 